\title{
Screening de primer trimestre de preeclampsia tardía en gestantes de bajo riesgo en un centro de bajo volumen obstétrico: validación externa de un modelo predictivo
}

\section{First trimester screening of late-onset preeclampsia in a low risk and low volume obstetrical setting: external validation of a predictive model}

\author{
D. Díaz Cobos, C. Laparte, A. Ruiz-Zambrana, J.L. Alcázar
}

\section{RESUMEN}

Fundamento. La validación externa de un modelo predictivo de predicción de preeclampsia tardía en un centro de bajo volumen obstétrico en gestantes de bajo riesgo obstétrico.

Métodos. Estudio prospectivo de 174 gestaciones únicas de $11^{+0}$ a $13^{+6}$ semanas de gestación en la Clínica Universidad de Navarra desde septiembre 2011 a marzo de 2013, que fue considerado como una cohorte de validación de un modelo descrito anteriormente para preeclampsia tardía en el hospital Clínic de Barcelona).

Resultados: Un total de 7 (4\%) mujeres desarrollaron PE tardía. En la cohorte de validación el área bajo la curva del modelo fue de 0,69 (IC del 95\% 0,45 a 0,93). Las tasas de detección para un 5, 10 y $15 \%$ de tasas de falsos positivos fueron $21,9,31,4$ y $38,6 \%$. Al comparar las áreas bajo la curva de la cohorte de validación con la cohorte de la construcción, no se encontraron diferencias estadísticamente significativas ( $\mathrm{p}=0,68)$.

Conclusión. La combinación de la historia clínica materna, la proteína placentaria A-asociada al embarazo y presión arterial media es moderadamente útil para predecir preeclampsia tardía en gestantes de bajo riesgo y en un centro de bajo volumen obstétrico. El modelo predictivo del hospital Clinic de Barcelona es una herramienta válida para predecir preeclampsia tardía en este entorno.

Palabras clave. Preeclampsia. Validación externa. Modelo predictivo. Gestante de bajo riesgo. Bajo volumen obstétrico.

\begin{abstract}
Background. The external validation of predictive model of late preeclampsia in a low volume and low risk obstetrical setting.

Methods. A cohort was created of 174 singleton pregnancies of $11^{+0}-13^{+6}$ gestational weeks at Clinica Universidad Navarra from September 2011 to March 2013, which was considered as a validation cohort of a previously described model for late PE (Hospital Clinic Barcelona).

Results. A total of 7 (4\%) women developed late PE. In the validation cohort the area under the curve of the model was 0.69 (95\% CI 0.45-0.93). Detection rates for a 5,10 and $15 \%$ of false positive rates were $21.9,31.4 \%$ and $38.6 \%$ respectively. When comparing the areas under the curve of the validation cohort with the construction cohort, no statistical differences were found $(\mathrm{p}=0.68)$.

Conclusion. The combination of maternal history, pregnancy associated plasma protein-A and mean arterial pressure is moderately useful to predict preeclampsia in a low risk and low volume obstetrical setting. The predictive model of the Clinic Hospital of Barcelona is a valid tool in predicting late preeclampsia in this setting.
\end{abstract}

Keywords. Preeclampsia. External validation. Predictive model. Low risk patients. Low obstetric setting.
Departamento de Obstetricia y Ginecología Clínica Universidad Navarra, Pamplona.

Recepción: 4 de julio de 2015

Aceptación provisional: 29 de septiembre de 2015

Aceptación definitiva: 16 de octubre de 2015

\author{
Correspondencia: \\ Daysi Díaz Cobos \\ Departamento de Obstetricia y Ginecología \\ Clínica Universidad Navarra, Pamplona. \\ Email: daysidiazcobos@yahoo.com
}

Los autores declaran que no existe ningún potencial conflicto de interés relacionado con el artículo. 


\section{INTRODUCCIÓN}

La preeclampsia (PE) es una causa importante de morbilidad-mortalidad materna y perinatal, que afecta aproximadamente al 3-5\% de las mujeres embarazadas en todo el mundo ${ }^{1-3}$. Debido a que la PE es una complicación perinatal grave, la detección temprana para identificar la población de alto riesgo antes de la aparición de los primeros síntomas sigue siendo un gran desafío clínico ${ }^{4}$.

Se han realizado muchos estudios de análisis de la historia clínica materna, marcadores bioquímicos y biofísicos y su capacidad de predicción de PE en el primer trimestre.

La historia clínica materna desempeña un papel importante en la identificación de mujeres con alto riesgo de PE. Un estudio presentó tasas de detección de PE precoz y tardía de 37,0 y $28,9 \%$, respectivamente, para una tasa de falsos positivos del $5 \% 5$. Otro estudio demostró que para una tasa de falsos positivos del $10 \%$ hubo un incremento en la detección de PE precoz del 47 al $76 \%$ y de un 41 al $52 \%$ para PE tardía cuando se combina la historia clínica con la determinación de la presión arterial teniendo más importancia la TA media que la sistólica o diastólica ${ }^{6}$.

En las mujeres que desarrollaron trastornos hipertensivos durante el embarazo las concentraciones séricas de proteína plasmática A asociada al embarazo (PAPP-A) estaban disminuidas en las que estaban de 11 y 13 + 6 semanas de gestación. En un estudio, se informó de que el uso de PAPP-A junto con marcadores biofísicos puede detectar más de dos tercios de PE precoz y un tercio de los casos de PE tardía cuando se clasifica el $2 \%$ de las gestaciones como de alto riesgo; tres cuartas partes de PE temprana se pudieron detectar con una tasa de falsos positivos del $5 \%{ }^{6,7}$.

El rendimiento de la presión arterial media en el primer trimestre ha demostrado una tasa de detección del $47 \%$ para PE tardía con una tasa de falsos positivos del $5 \%$, lo que aumenta a $76 \%$ cuando se combina con factores maternos ${ }^{8}$.

Las tasas de detección respectivas para PE tardía aumentaron del 41 al $52 \%$ y para la hipertensión gestacional del 31 al $48 \%{ }^{8}$.
Del mismo modo, el uso del Doppler de las arterias uterinas en el primer trimestre de embarazo como un medio no invasivo de la invasión trofoblástica, tiene una tasa de detección para PE precoz (con un 10\% de falsos positivos) del $47 \%$ cuando se utiliza individualmente, comparado con una tasa de detección del $81 \%$ cuando se combina con factores maternos. Las tasas de detección respectivas para PE tardía aumentaron de 41 a $45 \% 9$.

En los últimos años, los esfuerzos se han dirigido a construir algoritmos combinando todos estos factores, presentando tasas de detección de alrededor de 90\% para PE precoz y $60 \%$ para PE tardía ${ }^{10}$.

El objetivo de este estudio es demostrar que la combinación de la historia clínica materna, la PAPP-A y el Doppler de las arterias uterinas puede predecir PE en gestantes de bajo riesgo en un centro de bajo volumen obstétrico y validar externamente el modelo predictivo de PE tardía del hospital Clinic de Barcelona ${ }^{11}$.

\section{MATERIAL Y MÉTODOS}

Se realizó este estudio prospectivo de cohortes incluyendo todas las gestaciones únicas entre $11^{+0}$ y $13^{+6}$ semanas de gestación que acudieron a la consulta prenatal en el primer trimestre de gestación al Departamento de Obstetricia de la Clínica Universidad de Navarra, desde septiembre de 2011 hasta marzo de 2013. Las pacientes no elegibles poseían edad gestacional mayor de 14 semanas (datación basada en la longitud cráneo-caudal ${ }^{12}$. Los criterios de exclusión fueron anomalías fetales, test combinado positivo para aneuploidías, aborto tardío y pérdida de seguimiento. El motivo por el cual se excluyeron a las pacientes con fetos afectos de aneuploidías fue por los falsos positivos que podrían detectarse secundarios a la PAPP-A sérica disminuida. Los marcadores bioquímicos de cromosomopatías, tales como la PAPP-A sérica baja, se presenta tanto en pacientes con riesgo de PE como en pacientes con riesgo de cromosomopatía. Todas las pacientes fueron plenamente informadas sobre la naturaleza del estudio y el consentimiento informado 
por escrito fue firmado por las que aceptaron participar. El estudio fue aprobado por el Comité de Ética de la Clínica Universidad de Navarra.

Se recogieron prospectivamente las características maternas y la historia médica materna en el momento de la primera ecografía de primer trimestre (11,0 a 13,6 semanas). Las variables incluidas en nuestro estudio fueron las siguientes: la historia clínica materna, el índice de pulsatilidad de la arteria uterina (IP AUt) y la PAPP-A.

Las características maternas recogidas fueron edad materna, etnia, peso y estatura. La historia médica materna incluye la paridad, la PE previa, hipertensión crónica, la trombofilia, diabetes pre-gestacional, nefropatías y enfermedades autoinmunes.

La historia clínica materna fue obtenida mediante un cuestionario en la visita prenatal entre las 11 y 14 semanas, dirigido al antecedente de PE previa (edad gestacional al momento del inicio, la paridad, la edad materna y las complicaciones neonatales), antecedentes personales patológicos.

Se midió el índice de pulsatilidad de arteria uterina(IP AUt). Se identificó la arteria uterina izquierda y derecha con mapeo de flujo color. Se hizo medición transabdominal ${ }^{13}$ de la misma y obtención del índice de pulsatilidad usando el Doppler pulsado de ambas arterias uterinas. Se calculó el promedio de ambas arterias. Se utilizó el ecógrafo Voluson E8 (General Electric Healthcare, Milwaukee, EE.UU.)

La presión arterial media (PAM) fue medida entre las 11 y las 14 semanas con un esfigmomanómetro de mercurio. Una enfermera del departamento realizó la medición de la presión arterial en la consulta externa de acuerdo con un procedimiento estándar. La PA se midió en un brazo con la paciente sentada y después de 5 minutos de descanso.

La PAPP-A sérica se cuantificó entre la 11 y la 13,6 semana de gestación. En el informe del laboratorio se utilizó la PAPP-A como valor expresado en múltiplos de la mediana (MoM).

La PE se definió como presión arterial sistólica $\geq 140 \mathrm{~mm} \mathrm{Hg} \mathrm{y} \mathrm{/} \mathrm{o} \mathrm{presión} \mathrm{arterial}$ diastólica $\geq 90 \mathrm{~mm} \mathrm{Hg}$ medida en al menos
2 ocasiones en un intervalo de 4 horas, en gestantes $>20$ semanas previamente normotensas + , proteinuria $>300 \mathrm{mg}$ en la orina recogida en 24 horas. La PE tardía se definió como los criterios anteriores que se producen después de las 34 semanas de gestación.

Los resultados fetales obtenidos fueron: edad gestacional al momento del parto, tamaño pequeño para la edad gestacional, definido como el peso fetal estimado (PFE) entre el percentil 3-10 con los parámetros normales de Doppler. Restricción del crecimiento intrauterino (RCIU) se definió como un PFE < percentil 3 o parámetros Doppler anormales IP AUt $>$ p95, IP ACM $<$ p5, IP arteria umbilical > p95, flujo reverso en la arteria umbilical o flujo diastólico ausente, IP ductus venoso (DV) > p95, DV reverso y PFE < percentil 10. También se registró el peso al nacer, APGAR a los 5 min y el pH de la arteria umbilical al nacer.

\section{Análisis estadístico}

Las variables categóricas se expresaron como frecuencias absolutas y relativas. Se empleó la prueba $\chi 2$ de Pearson o la prueba exacta de Fisher para comparar las variables categóricas o cualitativas entre los grupos. Se confirmó la distribución normal de las variables continuas mediante el test de Kolmogorov-Smirnov. Las variables de distribución normal se presentaron como media (SD) y las variables de distribución no normal se presentaron como mediana (rango). Se usó la prueba t de Student o prueba U de Mann Whitney para analizar las diferencias de las variables continuas entre los grupos.

Se utilizó un modelo de regresión logística para estimar el riesgo individual $a$ prio$r i$ con respecto a las siguientes variables: historia personal de diabetes; hipertensión arterial crónica; enfermedad renal o autoinmune; trombofilia congénita o adquirida; antecedente de preclampsia (PE) previa; edad materna; IMC $\left(\mathrm{kg} / \mathrm{m}^{2}\right)$; tabaquismo (cigarrillos/día); etnia y paridad.

Se realizó análisis de regresión logística para estimar el riesgo individual para desarrollar PE precoz o tardía con respecto a las siguientes variables: riesgo a priori (transformación log), log MoM IP-AUt; log 
MoM PAPP-A y log MoM PAM. Se construyeron curvas para analizar el desempeño del modelo. Este análisis se expresó como tasa de detección para los diferentes puntos de corte de tasas de falsos positivos.

Para la predicción de la PE tardía se realizó un análisis de la curva ROC con suavizado binormal ${ }^{14}$ y el modelo lineal se montó en los cuartiles de las sensibilidades y especificidades.

Las curvas ROC suavizadas de la cohorte de construcción y validación fueron comparadas por unpairly bootstrapping de 2.000 repeticiones de cada curva ${ }^{15}$.

Se utilizó la versión de software estadístico R 2.15.1 (Fundación para la Computación de Estadística R; http://www.r-project. org/foundation) con la versión del paquete pROC 1.7.2 para realizar todos los análisis estadísticos.

\section{RESULTADOS}

Se estudiaron un total de 200 pacientes que fueron atendidas dentro del periodo de estudio y cumplieron los criterios de elegibilidad. De ellas, 26 fueron excluidas debido a anomalías fetales, test de cribado combinado positivo para aneuploidías, aborto tardío y pérdida de seguimiento.

Entre las 174 pacientes analizadas 8 (4,6\%) desarrollaron PE: 7 desarrollaron PE tardía $(87,5 \%)$ y una paciente presentó $\mathrm{PE}$ precoz $(12,5 \%)$. La tabla 1 muestra las características epidemiológicas y clínicas de la población estudiada.

En cuanto a los datos perinatales, la mediana de edad gestacional al momento del parto fue de 39,9 semanas (33-41 semanas) y el peso promedio al nacimiento fue de 3.366 gramos (rango: 2.100 a 4.700 gramos).

Tabla 1. Características epidemiológicas y características clínicas de la población estudiada clasificada en grupos

\begin{tabular}{|c|c|c|c|}
\hline Variable & No afectada $(n=166)$ & PE $(n=8)$ & $\mathbf{p}<\mathbf{0 , 0 0 5}$ \\
\hline Edad (a), mediana (IQR) & $34(23-44)$ & $30,88(28-35)$ & 0,025 \\
\hline IMC kg/m², mediana (IQR) & $22,9(16,59-33,75)$ & $24,38(19-35)$ & 0,179 \\
\hline \multicolumn{4}{|l|}{ Etnia; n\% } \\
\hline Caucásica & $159(95,8 \%)$ & $6(75 \%)$ & 0,009 \\
\hline Negra & $0(0 \%)$ & $0(0 \%)$ & - \\
\hline Latino americana & $5(3,1 \%)$ & $2(25 \%)$ & 0,020 \\
\hline Otras & $2(1,1 \%)$ & $0(0 \%)$ & 0,087 \\
\hline \multicolumn{4}{|c|}{ Antecedentes personales patológicos; n\% } \\
\hline Hipertensión crónica & $1(0,6 \%)$ & $1(12,5 \%)$ & 0,091 \\
\hline Diabetes mellitus & $1(0,6 \%)$ & $0(0 \%)$ & - \\
\hline Nefropatía & $0(0 \%)$ & $0(0 \%)$ & - \\
\hline Trombofilias & $6(3,6 \%)$ & $1(12,5 \%)$ & 0,288 \\
\hline \multicolumn{4}{|l|}{ Antecedentes obstétricos, n\% } \\
\hline Nuliparidad & $53(31,9 \%)$ & $3(37,5 \%)$ & 0,510 \\
\hline PE previa & $5(3 \%)$ & $1(12,5 \%)$ & 0,251 \\
\hline PAM, mm Hg, mediana (IQR) & $75,94 \mathrm{~mm} \mathrm{Hg}(60-117)$ & 81,38 mm Hg (71-93) & 0,056 \\
\hline IP AUt, mediana(IQR) & $1,46(0,56-3,6)$ & $1,58(0,9-2,5)$ & 0,480 \\
\hline PAPP-A sérica (MoM), mediana (IQR) & $1,22 \mathrm{MoM}(0,29-3,55)$ & $0,91 \mathrm{MoM}(0,31-1,43)$ & 0,220 \\
\hline
\end{tabular}

PAM: Presión arterial media; IP AUt: Indice de pulsatilidad arteria uterina; PAPP-A: Proteína placentaria asociada al embarazo; IQR: rango intercuantil 
Se realizó cesárea al 22,7\% de nuestras pacientes. Se midió el pH de los vasos umbilicales, 17 (9,9\%) tuvieron un $\mathrm{pH}$ umbilical al nacer por debajo de 7,20 y 5 (2,9\%) tenían una puntuación de 5 min de APGAR por debajo de 7 .

Las mujeres que desarrollaron PE fueron más jóvenes (media de 30,9 frente a 34; $\mathrm{p}=0,244)$. No se encontró asociación entre la aparición de PE en la actual gestación y el antecedente de PE. Las mujeres con PE tenían una edad gestacional menor al momento del parto 37,9 años [DS 1,45] frente a 39,2 años [DS 1,44]; $\mathrm{p}=0,016)$. Hubo 6 casos de restricción de crecimiento intrauterino (RCIU), 2/8 (25\%) en el grupo PE y $4 / 166$ (2,4\%) en el grupo no afectado.

Los valores medios de IP AUt (11 a 13,6 semanas) y PAPP-A sérica no difirieron significativamente en las mujeres que desarrollaron PE. Se encontró una tendencia hacia valores más altos de la PAM tomada en primer trimestre en las mujeres que desarrollaron PE en comparación con las gestantes que no presentaron PE $(81,4$ vs $75,9 ; \mathrm{p}=0,056)$. Las figuras 1-3 muestran la distribución de IP AUt, PAM y PAPP-A sérica asociada a la presencia de PE en general.
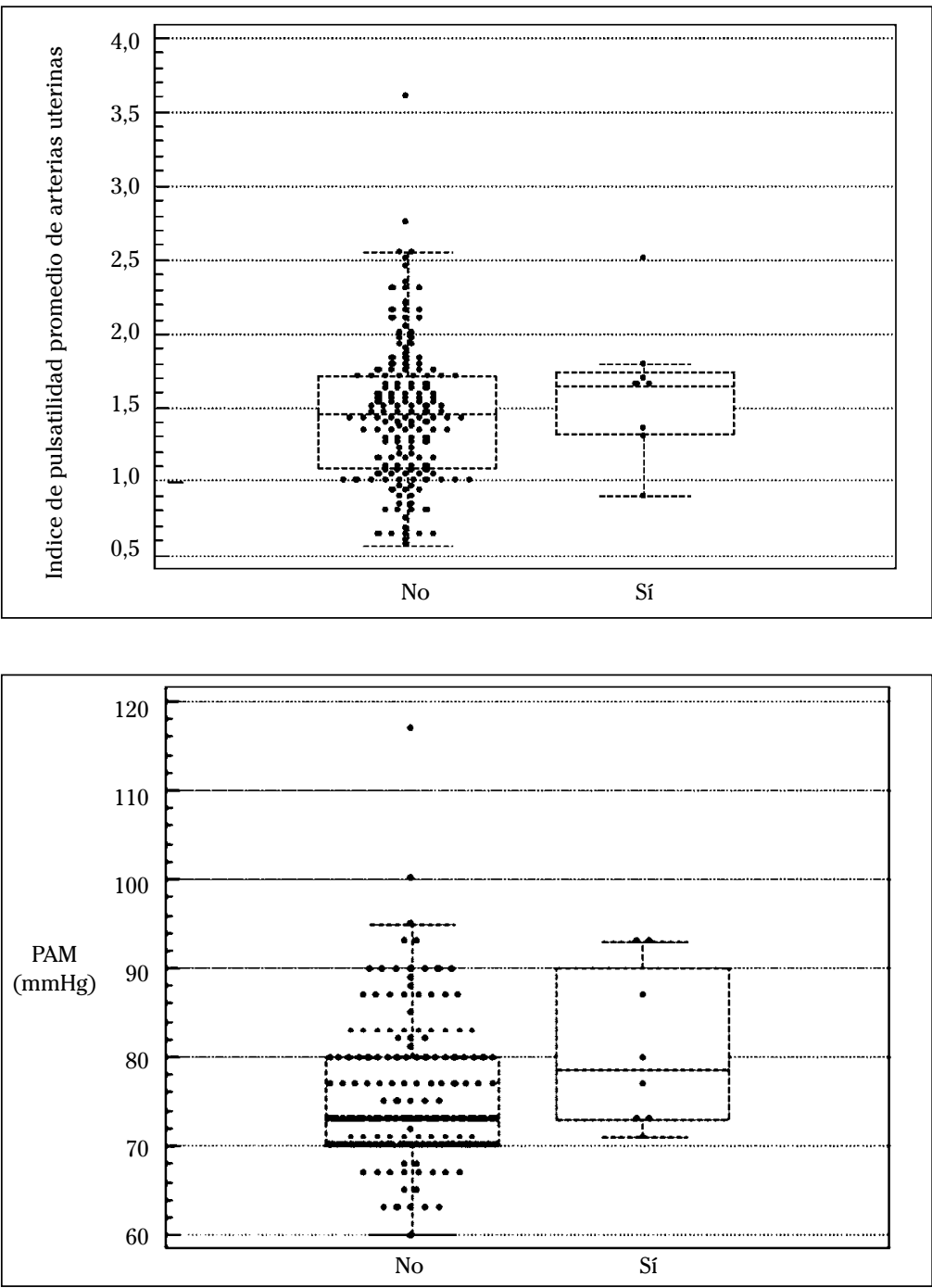

Figura 1.

Distribución de la media del Indice de Pulsatilidad de Arterias Uterinas en el primer trimestre en pacientes con PE.

\section{Figura 2.}

Distribución de la media de la Presión Arterial Media(PAM) entre la $11^{+0}-13^{+6}$ semanas en pacientes con PE. 
Figura 3.

Distribución de los niveles de PAPP-A sérica en el primer trimestre en pacientes con $\mathrm{PE}$

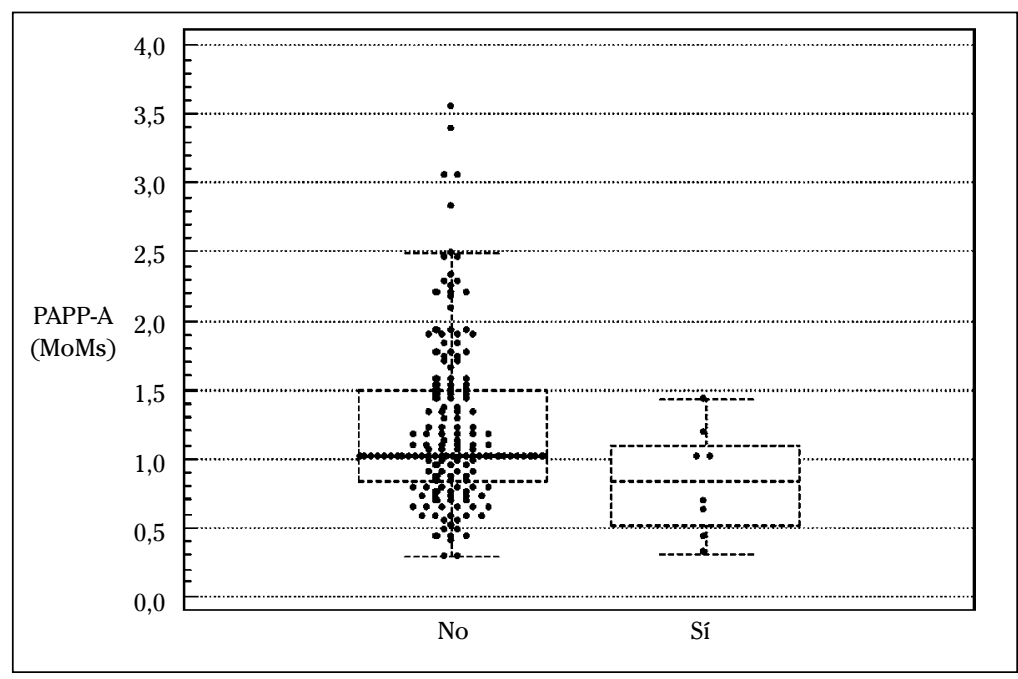

Se utilizó un modelo de regresión logística para predecir un riesgo a priori para $\mathrm{PE}$ precoz y PE tardía [riesgo a priori $=\mathrm{e}^{\mathrm{y}}$ $\left./\left(1+\mathrm{e}^{\mathrm{y}}\right)\right]$, donde e es el error y la Y es la probabilidad:

- PE tardía Y = -6,135_ (2,124 si PE anterior) _ (1,571 si hipertensión crónica) - (0,958 si diabetes mellitus) - (1,416 si trombofilia) - $(0,487$ si multípara $)$ $(0,093$ * índice de masa corporal).

- PE precoz Y = -7,703 (0,086 * índice de masa corporal) - (1,708 si hipertensión crónica $)$ - (4,033 si enfermedad renal) - (1,931 si paridad, $\mathrm{PE}$ anterior) - $(0,005 \text { si paridad, sin PE anterior })^{11}$.

Los siguientes modelos se ajustan mejor al riesgo a posteriori específico en la paciente (un riesgo a posteriori $=\mathrm{e}^{\mathrm{y}} /\left[1+\mathrm{e}^{\mathrm{y}}\right]$ ):

- PE tardía $\mathrm{Y}=0,328(2.205 *$ ingrese un riesgo a priori $)-(1,307 * \log \mathrm{MoM}$ PAPP-A)

- PE precoz $\mathrm{Y}=-0,320(2.681$ * ingrese un riesgo a priori $)-(13,132 * \log \mathrm{MoM}$ significa IP AUt $)-(25,733$ * $\log$ MoM MAP) ${ }^{11}$.

Se construyó una curva ROC para PE tardía (con exclusión de un caso de aparición temprana) y se comparó con la curva obtenida de la cohorte original donde se construyó el modelo. En la cohorte de validación el área bajo la curva del modelo fue de 0,69 (IC del 95\% 0,45 a 0,93), mien- tras que en la cohorte original era 0,71 (IC del 95\% 0,66 a 0,76). Las tasas de detección para un 5,10 y $15 \%$ de falsos positivos fueron $21,9,31,4$ y $38,6 \%$. Al comparar las áreas bajo la curva de la cohorte de validación con la cohorte de la construcción, no se encontraron diferencias estadísticamente significativas $(\mathrm{p}=0,68)$. La figura 4 demuestra las dos curvas ROC.

\section{DISCUSIÓN}

El objetivo de nuestro estudio fue establecer el riesgo predictivo de PE basado en parámetros clínicos, biofísicos y bioquímicos en gestantes de bajo riesgo en un centro de bajo volumen obstétrico. Se han estudiado estos parámetros entre la $11^{+0}-13^{+6}$ semanas.

Se han realizado muchos estudios analizando el desempeño de la historia clínica materna, marcadores bioquímicos y biofísicos y su capacidad de predicción de PE en primer trimestre. La historia clínica materna desempeña un papel importante en la identificación de mujeres con alto riesgo de PE. Un estudio presentó tasas de detección de PE precoz y tardía de 37,0 y 28,9\%, respectivamente, para una tasa de falsos positivos del $5 \%$.

Se hizo una anamnesis dirigida, y se obtuvo una tasa global de PE del 4,6\% siendo la tasa de PE tardía más alta que la PE 


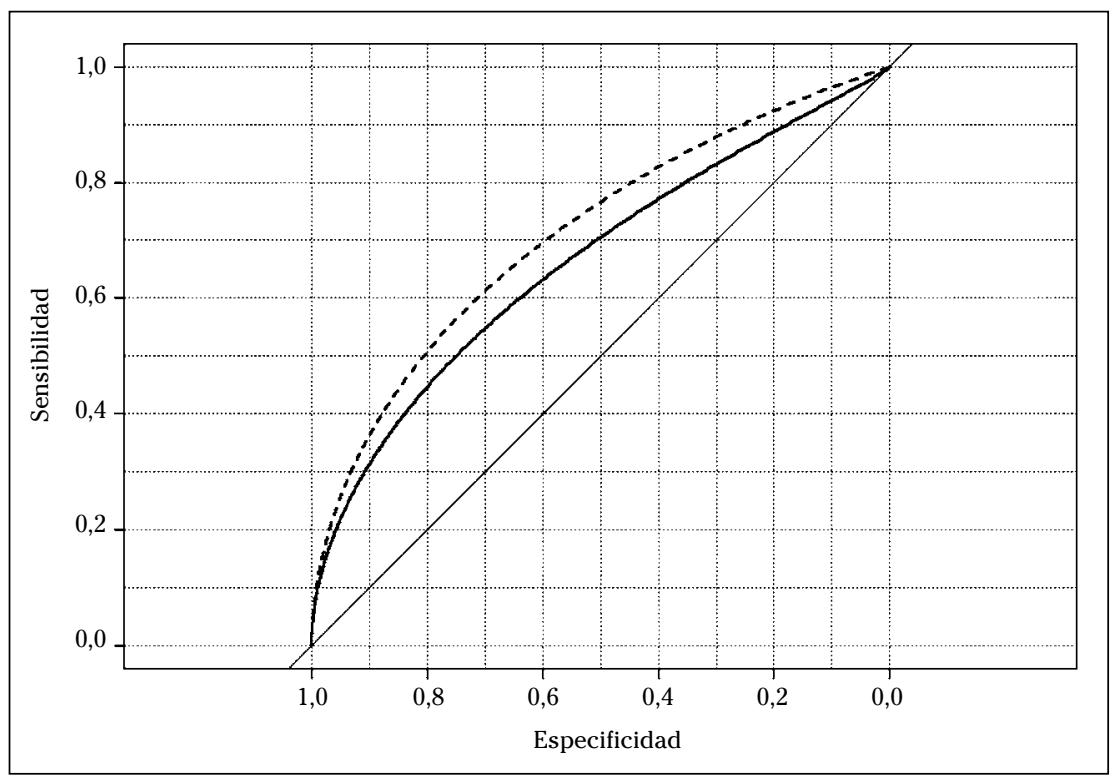

Figura 4. Curvas ROC para PE en las cohortes de construcción (--) y validación (-).

temprana. Estos hallazgos se correlacionan con los datos encontrados en la literatura en la cual la prevalencia de PE tardía es significativamente mayor que la de PE precoz. El desarrollo de PE tardía se cree que es influenciado por predisposición materna con escaso componente placentario ${ }^{2,3}$.

Entre nuestras gestantes, el $0,6 \%$ presentaban DM pre-gestacional discrepando con la prevalencia general de esta última del 2-5\% en gestante ${ }^{10}$.

Basado en las características clínicas encontramos un 1,2\% de pacientes con HTA crónica con edad media de 33 años, dato que difiere con respecto a la prevalencia general de HTAC en embarazo en gestantes de 30-39 años ${ }^{16}$, resultado probablemente secundario al bajo volumen obstétrico.

En nuestra población no se ha hallado asociación entre la nuliparidad y la aparición de PE, dato que contrasta con la asociación importante entre estos dos últimos descritos en la literatura ${ }^{17}$, lo que puede atribuirse a que la mayoría de nuestras pacientes eran multíparas con período intergenésico menor a 10 años y sin factores coexistentes que aumentaran su riesgo a presentar PE.
No se ha encontrado asociación entre el IMC y PE, ya que el IMC encontrado en nuestra población fue de $23 \mathrm{y}$ el IMC descrito en la literatura como factor predisponente a presentar PE es de $30^{18}$. Tampoco ha aparecido asociación entre PE tardía y antecedente de PE previa, HTAC, DM pregestacional, trombofilias y TRA con respecto a lo publicado en que se demuestra la asociación de estas condiciones preexistentes con la aparición de PE precoz y no $\operatorname{tardía}^{17,19,20-22,23}$.

La literatura demuestra asociación de trombofilias con la aparición de PE precoz o severa ${ }^{24}$ dato que puede explicar la ausencia de asociación con nuestros casos de PE tardía.

En un estudio, se informó de que el uso de PAPP-A junto con marcadores biofísicos puede detectar más de dos tercios de PE precoz y un tercio de los casos de PE tardía cuando se clasifica el $2 \%$ de las gestaciones como de alto riesgo; tres cuartas partes de PE precoz se pudieron detectar con tasa de falsos positivos del $5 \%$. En nuestro estudio los valores del IP AUt $\left(11^{+0}-13^{+6}\right)$ y PAPP-A sérica no mostraron individualmente asociación con la aparición de PE tardía. 
En nuestro estudio no observamos asociación entre el IP AUt con PE tardía. El IP AUt se relaciona con una placentación inadecuada, que puede explicar la aparición de PE precoz y no tardía, ya que esta última es de etiología multifactorial más que placentaria. El Doppler de las arterias uterinas en primer trimestre tiene una tasa de detección para PE precoz (con un 10\% de falsos positivos) del $81 \%$ cuando se combina con factores maternos. Las tasas de detección respectivas para $\mathrm{PE}$ tardía aumentaron del 41 a $45 \%{ }^{9}$. En los últimos años se han construido algoritmos que combinan todos estos factores, presentando tasas de detección de alrededor de $90 \%$ para PE precoz y $60 \%$ para PE $\operatorname{tardía}^{10}$. Nuestro trabajo no ha podido demostrar asociación entre el IP AUt y la PE tardía. Con nuestros resultados hemos realizado la validación externa del modelo predictivo de PE propuesto por el hospital Clinic de Barcelona, respecto a la PE tardía, ya que solo obtuvimos un caso de PE temprana.

Antes de considerar si se debe utilizar un modelo de predicción clínica, se requiere que su rendimiento predictivo haya sido evaluado en los conjuntos de datos que no se utilizaron para construir el modelo, o sea realizar una validación externa. Externamente la validación del modelo en un entorno geográfico diferente es una buena medida de su generalización y exportabi$\operatorname{lidad}^{25}$. Muy pocos estudios han validado externamente modelos de primer trimestre. Recientemente, el rendimiento de un algoritmo desarrollado en el Reino Unido ${ }^{26}$ se ha evaluado en una población australia$\mathrm{na}^{27}$, mostrando una reducción en la tasa de detección del 83 al 41,7\% con una tasa de falsos positivos de $5 \%$ para la PE precoz, aunque el pequeño número de casos en la cohorte de validación y las diferencias en las características de la población o las técnicas de medición no permiten sacar conclusiones firmes sobre esta diferencia.

Nuestro estudio apoya la validación externa de un modelo previamente construido de PE tardía en una población diferente de bajo riesgo en un centro de bajo volumen obstétrico, aunque el rendimiento es solo moderado en términos de tasa de detección. Las tasas de detección para un 5,10 y $15 \%$ de falsos positivos fueron 21,9 , 31,4 y $38,6 \%$. Al comparar las áreas bajo la curva de la cohorte de validación con la cohorte de la construcción, no se encontraron diferencias estadísticamente significativas $(\mathrm{p}=0,68)$. Desafortunadamente, el tamaño de nuestra muestra es muy pequeña, haciendo difícil sacar conclusiones a partir de las curvas ROC.

La prevalencia de PE tardía es significativamente mayor que la de PE precoz. El desarrollo de PE tardía se cree que está influenciada significativamente por la predisposición materna con poco o insignificante componente placentario, lo que explica una menor presencia de restricción de crecimiento intrauterino (RCIU) que se produce en la mayoría de los casos de PE precoz; esto hace que la predicción de PE tardía en el primer trimestre sea bastante desafiante ${ }^{28}$. En el presente estudio, las mujeres con PE tenían una edad gestacional menor al momento del parto 37,9 [DS 1,45] frente a 39,2 [DS 1,44]; $p=0,016$, lo que refleja una mayor incidencia de la inducción del parto debido a la aparición de PE como criterio de finalización de la gestación. Los beneficios de la predicción de PE tardía se sustentan indirectamente por un ensayo clínico aleatorio que demuestra que la inducción electiva a las 37 semanas mejora los resultados maternos sin ningún empeoramiento en los resultados neonatales ${ }^{29}$.

Reconocemos que una limitación importante de nuestro estudio es el tamaño de la muestra debido a que se desarrolló en un centro privado con bajo volumen obstétrico y gestantes de bajo riesgo; esto no nos permitió poder analizar el rendimiento del modelo para predecir la PE precoz. Recientemente, se ha publicado otro algoritmo por el mismo grupo que incluía factores angiogénicos en primer trimestre, que muestra una mejoría relevante en la tasa de detección de PE tardía (69\% con una tasa de falsos positivos del $10 \%)^{30}$. Lamentablemente, carecemos de datos sobre los factores angiogénicos en el algoritmo para probar la validez de este modelo.

Concluimos que la combinación de la historia clínica materna, la PAPP-A sérica 
y la PAM medida en primer trimestre es moderadamente útil para predecir $\mathrm{PE}$ en gestantes de bajo riesgo obstétrico en un centro de bajo volumen.

Consideramos que el modelo predictivo del hospital Clinic de Barcelona es una herramienta válida para predecir la aparición de PE tardía en centros de bajo volumen obstétrico.

\section{BIBLIOGRAFÍA}

1. Poon L, Akolekar R, Lachmann R, Beta J and NiCOLAIDES K. Hypertensive disorders in pregnancy: screening by biophysical and biochemical markers at 11-13 weeks. Ultrasound Obstet Gynecol 2010; 35: 662-670.

2. James J, Whitley G. Pre-eclampsia: fitting together the placental, immune and cardiovascular pieces. J Pathol 2010; 221: 363-378.

3. Cerdeira A, Karumanchi A. Biomarkers in preeclampsia. Biomarkers in kidney disease 2010; 11: 385-426.

4. Poprawski G, Wender-Ozegowska E, Zawiejska A, BRAZERT J. Modern methods of early screening for preeclampsia and pregnancy induced hypertension a review. Ginekol Pol 2012; 83: 688-693.

5. Poon L, Kametas N, Maiz N, Akolekar R, NicolaiDES K. First-trimester prediction of hypertensive disorders in pregnancy. Hypertension 2009; 53: 812-818.

6. Poon lC, Kametas NA, Valencia C, Chelemen T, Nicolaides KH. Hypertensive disorders in pregnancy: screening by systolic diastolic and mean arterial pressure at 11-13 weeks. Hypertens Pregnancy 2011; 30: 93-107.

7. Cuckle HS. Screening for Pre-eclampsia-lessons from aneuploidy screening. Placenta 2011; 32: 42-48.

8. Poon L, Kametas NA, Chelemen T, Leal A, NicoLAIDES KH. Maternal risk factors for hypertensive disorders in pregnancy: a multivariate approach risk for hypertensive disorders. J Human Hypertension 2010; 24: 104-110.

9. Poon LCy, Staboulidou I, Maiz N, Plasencia W, NiCOLAIDES KH. Hypertensive disorders in pregnancy: screening by uterine artery doppler at 11-13 weeks. Ultrasound Obstet Gynecol 2009; 34: 142-148.

10. Poon lC, Karagiannis G, Leal A, Romero XC, NiCOLAIDES KH. Hypertensive disorders in pregnancy: screening by uterine artery doppler imaging and blood pressure at 11-13 weeks. Ultrasound Obstet Gynecol 2009; 34: 497-502.
11. Scazzocchio E, Figueras F, Crispi F, Meler E, Masoller N, Mula R et al. Performance of a first-trimester screening of preeclampsia in a routine care low-risk setting. Am J Obstet Gynecol 2013; 208: 203.e1-203.e10.

12. Robinson HP, Fleming J. A critical evaluation of sonar "crown-rump length" measurements. BJOG: An International Journal of Obstetrics \& Gynaecology 1975; 82: 702-710.

13. Hollis B, Mavrides E, Campbell S, Tekay A, ThilaGANATHA B. Reproducibility and repeatability of transabdominal uterine artery doppler velocimetry between 10 and 14 weeks of gestation. Ultrasound obstetrics gynecology 2001; 18: 593-597.

14. Hanley J. The robustness of the binormal assumptions used in fitting ROC curves. Med Decis Making 1988; 8: 197-203.

15. Delong E, Delong DM, Clarke-Pearson D. Comparing the areas under two or more correlated receiver operating characteristic curves: a nonparametric approach. Biometrics 1988; 44: 837-845.

16. SIBAI BM. Chronic hypertension in pregnancy. Obstet Gynecol 2002; 100: 369-377.

17. Barton J, Sibai B. Prediction and prevention of recurrent preeclampsia. Fetal Diagn Ther 2008; 112: 359-372.

18. Catov J, Ness R. Risk of early or severe preeclampsia related to preexisting condition. Int J Epidemiol 2007; 36: 412-419.

19. Frishman W, Schlocker S. Pathophysiology and medical management of systemic hypertension in pregnancy. Cardiology Rev 2005; 13 : 274-284.

20. Prado D, Piovesan D. Association of anticardiolipin antibodies with preeclampsia: a systematic review and meta-analysis. Obstet Gynecol 2010; 116: 1433-1443.

21. Branch W, Fuint DT. Antiphospholipid antibodies in women at risk for preeclampsia. Am J Obstet Gynaecol 2001; 184: 825-834.

22. Hernández-Diaz S, Toh S. Risk of preeclampsia in first and subsequent pregnancies: prospective cohort study. BMJ 2009; 338: b2225.

23. Hawfield A, Freedman B. Pre-eclampsia:the pivotal role of the placenta in its pathophysiology and markers of early detection. Ther Adv Cardiovasc Dis 2009; 3: 65-73.

24. Lin J, August P. Genetic thrombophilias and preclampsia: a meta-analysis. Amer College Obstet Gynaecol 2005; 105: 182-192.

25. Altman DG, Vergouwe Y, Royston P, Moons KG. Prognosis and prognostic research: validating a prognostic model. BMJ 2009; 338: 14321435 . 
26. Akolekar R, Syngelaki A, Poon L, Wright D, NiCOLAIDES KH. Competing risks model in early screening for preeclampsia by biophysical and biochemical markers. Fetal Diagn Ther 2013; 33: 8-15.

27. Park F, Leung C, Poon LCY, Williams P, Rothwell $\mathrm{S}$, HyetT J. Clinical evaluation of a first trimester algorithm predicting the risk of hypertensive disease of pregnancy. Australian New Zealand J Obstet Gynaecol 2013; 53: 532-539.

28. Crispi F, Dominguez C, Llurba E, Martin-Gallan P, Gratacos E. Placental angiogenic growth factors and uterine artery doppler findings for characterization of different subsets in preeclampsia and in isolated intrauterine growth restriction. Am J Obstet Gynecol 2006; 195: 201-207.

29. Koopmans CM, Bijlenga D, Groen H, ViJgen SM, AARnoudse JG, BEKEDAM DJ et al. HYPITAT study group. Induction of labour versus expectant monitoring for gestational hypertension or mild pre-eclampsia after 36 weeks gestation (HYPITAT): a multicentre, open-label randomised controlled trial. Lancet 2009; 374: 979988.

30. Crovetto F, Figueras F, Triunfo S, Crispi F, RodriGuez-Sureda V, Peguero A et al. Added value of angiogenic factors for the prediction of early and late preeclampsia in the first trimester of pregnancy. Fetal Diagn Ther 2014; 35: 258-266. 